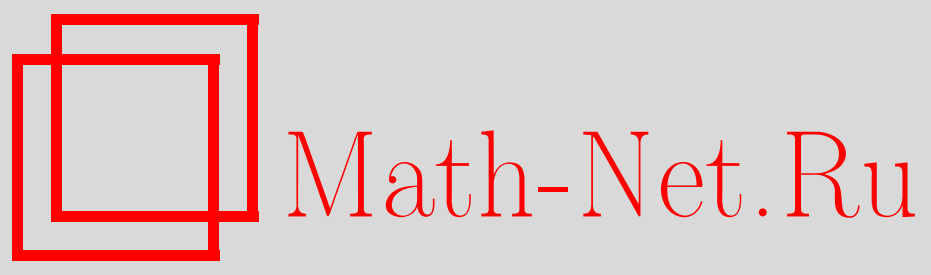

Хианглин Вей, Случай существования внутренних точек трех типов, Матем. заметки, 2013, том 93, выпуск 1, 152-156

DOI: https://doi.org/10.4213/mzm10138

Использование Общероссийского математического портала Math-Net.Ru подразумевает, что вы прочитали и согласны с пользовательским соглашением http://www . mathnet.ru/rus/agreement

Параметры загрузки:

IP : 54.80 .97 .219

26 апреля 2023 г., 14:21:04

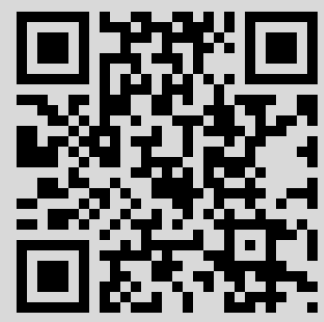




\section{Случай существования внутренних точек трех типов}

\section{Хианлин Вей}

1. Введение. В данной работе рассматривается только плоское точечное множество в общем положении, т.е. точечное множество, в котором никакие три точки не являются коллинеарными (т.е. не лежат на одной прямой). В 2001 г. Авис, Хосоно и Урабе [1] исследовали следующую задачу, связанную с наиболее знаменитой и привлекательной задачей Эрдеша-Секереша.

Для любого челого числа $k \geqslant 1$ найти наименьшее челое число $g(k)$ такое, что всякое точечное множество $P$, содержащее по крайней мере $g(k)$ внутренних точек, имеет подмножество, содержащее в точности $k$ внутренних точек.

В работе [1] показано, что

$$
g(1)=1, \quad g(2)=4, \quad g(3) \geqslant 8 .
$$

Февенс [2] показал, что

$$
g(k) \geqslant 3 k-1 \quad \text { для } k \geqslant 3 .
$$

Недавно мы улучшили оценку нижней грани, доказав, что

$$
g(k) \geqslant 3 k \quad \text { при } \quad k \geqslant 3
$$

в [3], и получили точное значение $g(3)=9$ (см. [4], [5]).

В 2000 году Авис, Хосоно и Урабе [6] рассматривали следующую задачу:

Для любого челого числа $k \geqslant 1$ найти наименьшее челое число $h(k)$ такое, что всякое точечное множество $P$, содержащее по крайней мере $h(k)$ внутренних точек, имеет подмножество, содержащее в точности $k$ или $k+1$ внутренних точек множества $P$.

Они привели единственные известные нетривиальные точные значения $h(k)$ :

$$
h(3)=3 \quad \text { и } \quad h(4)=7 .
$$

Февенс [2] доказал, что

$$
h(k) \geqslant 2 k+1 \quad \text { при } 5 \leqslant k \leqslant 8 \quad \text { и } \quad h(k) \geqslant 3 k-7 \text { при } k \geqslant 8 .
$$

Эти задачи привлекли наше внимание, и в данной работе мы рассматриваем задачу отыскания наименьшего целого числа $t(k)$ такого, что всякое точечное множество $P$, содержащее по крайней мере $t(k)$ внутренних точек, имеет подмножество, содержащее в точности $k$ или $k+1$ или $k+2$ внутренних точек множества $P$. Решение этой задачи может быть полезно для отыскания $g(k)$.

Работа выполнена при поддержке Национального фонда естественных наук Китая (10901045), Фонда естественных наук провинции Хебей (А2010000828), Научного фонда университета науки и технологии провинции Хебей (QD200955) и Фонда естественных наук Департамента образования провинции Хебей (Z2009113). На заключительной стадии данной работы, автор работал в Пекинском университете, Китай, и получил финансовую поддержку от Пекинском университета.

DOI: $10.4213 / \mathrm{mzm} 10138$

(C) ХиАнлин Вей, 2013 
2. Определения и обозначения. Напомним некоторые определения и обозначения, которые использовались в работе [4].

Пусть $P$ - точечное множество, $C H(P)$ - выпуклая оболочка множества $P$, а $V(P)=$ $\left\{v_{1}, v_{2}, \ldots, v_{m}\right\}-$ множество вершин выпуклой оболочки $C H(P)$, или, коротко, множества $P$. Здесь вершины $v_{1}, v_{2}, \ldots, v_{m}$ всегда отсчитываются против часовой стрелки. Внутренняя точка множества $P$ - это точка множества $P$, которая не лежит на границе выпуклой оболочки $C H(P)$. Через $I(P)$ обозначим совокупность внутренних точек множества $P$. При индексации множества, состоящего из $t$ точек, индексы отождествляются по модулю $t$. Пусть $Q \subset P$. Будем говорить, что $Q$ содержит внутреннюю точку $p$ множества $P$, если $p \in I(P) \cap \operatorname{int} C H(Q)$, где int $C H(Q)$ - внутренность выпуклой оболочки множества $Q$, и для краткости будем писать $I^{*}(Q)$ вместо $I(P) \cap \operatorname{int} C H(Q)$. Легко привести примеры, показывающие, что $I(Q) \neq I^{*}(Q)$. Через $i^{*}(Q)=\left|I^{*}(Q)\right|$ обозначим число внутренних точек множества $P$, содержащихся в $Q$. Если $i^{*}(Q)=k$, то будем говорить, что $Q$ - “подмножество, содержащее в точности $k$ внутренних точек множества $P$ " или, для краткости, "подмножество, содержащее в точности $k$ внутренних точек". Будем также говорить, что точечное множество $Q$ находится в выпуклом положении или является выпуклым, если оно образует множество вершин некоторого точечного множества. Применяя эти определения, можно записать $t(k)$ следующим образом:

$t(k)=\min \left\{s:|I(P)| \geqslant s \rightarrow \exists Q \subset P, i^{*}(Q)=|I(P) \cap \operatorname{int} C H(Q)|=k\right.$, или $k+1$, или $\left.k+2\right\}$.

Множество точек $T$ называется треугольником и обозначается как $T=\triangle v_{1} v_{2} v_{3}$, если $V(T)=\left\{v_{1}, v_{2}, v_{3}\right\}$. Внутренняя точка треугольника $T$ называется $(x, y)$-разделителем треугольника $T$, если она разделяет $T$ на три треугольника, содержащих $x \geqslant y \geqslant 0$ внутренних точек, соответственно. Через $H(a b ; c)$ обозначим полуплоскость, ограниченную прямой линией $a b$ так, что точка $c$ лежит внутри полуплоскости. Множество точек $P$ называется дефицитным точечным множеством $P(m, s, k)$, если $|V(P)|=m,|I(P)|=s$ и для любого $Q \subset P$ выполняются неравенства $i^{*}(Q) \neq k, k+1, k+2$.

3. Нижняя грань для $t(k)$. Пусть $k \geqslant 6$ - целое число. Представим дефицитное точечное множество $P(3,(3 k-1) / 2, k)$ для нечетного $k$ (см. рис. 1$)$, где

$$
\begin{array}{lll}
\left|V\left(P \backslash\left\{v_{1}\right\}\right)\right|=\frac{k-3}{2}+4 & \text { при } & i^{*}\left(P \backslash\left\{v_{1}\right\}\right)=k-1, \\
\left|V\left(P \backslash\left\{v_{2}\right\}\right)\right|=\frac{k-1}{2}+4 & \text { при } & i^{*}\left(P \backslash\left\{v_{2}\right\}\right)=k-2, \\
\left|V\left(P \backslash\left\{v_{3}\right\}\right)\right|=\frac{k-3}{2}+4 & \text { при } & i^{*}\left(P \backslash\left\{v_{3}\right\}\right)=k-1,
\end{array}
$$

и дефицитное точечное множество $P(3,3 k / 2, k)$ для четного $k$ (см. рис. 2$)$, где

$$
\left|V\left(P \backslash\left\{v_{i}\right\}\right)\right|=\frac{k-2}{2}+4 \quad \text { при } \quad i^{*}\left(P \backslash\left\{v_{i}\right\}\right)=k-1,
$$

где $i=1,2,3$. Таким образом, известно, что

$$
t(k) \geqslant \frac{3 k+1}{2} \text { для нечетного } k, \quad \text { и } \quad t(k) \geqslant \frac{3 k}{2}+1 \text { для четного } k \text {. }
$$

4. Доказательство равенства $t(6)=10$.

Лемма 1 [1]. Всякое плоское точечное множество, содержащее по крайней мере $k$ внутренних точек, имеет выпуклое подмножество, содержащее от $k$ до $\lfloor 3 k / 2\rfloor$ внутренних точек.

Из леммы 1 следует, что $t(k)=k$ при $1 \leqslant k \leqslant 5$.

Для доказательства равенства $t(6)=10$ нам понадобится следующее свойство дефицитного точечного множества $P(3,9,6)$. 


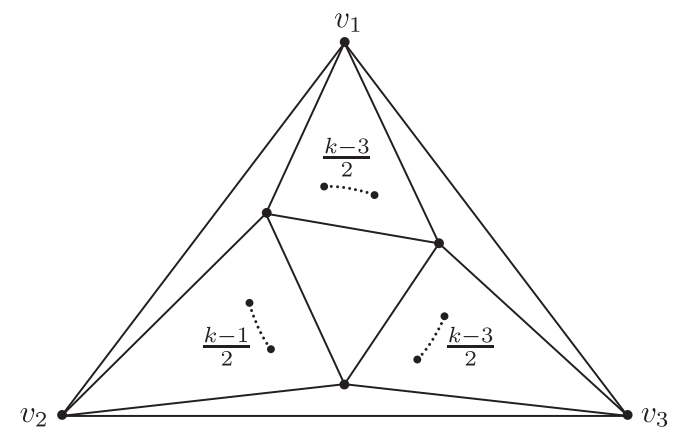

Рис. 1. $k$ нечетное

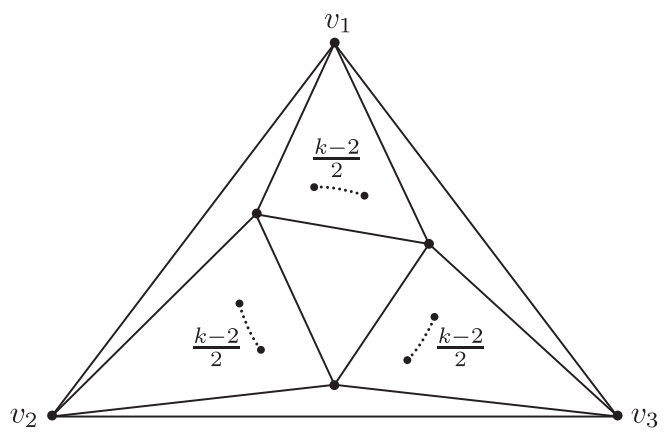

Рис. 2. $k$ четное

Лемма 2. Пусть $P$ - дефииитное точечное множество $P(3,9,6)$, где $V(P)=\left\{v_{1}\right.$, $\left.v_{2}, v_{3}\right\}$. Тогда Р обладает следующими свойствами:

$$
\left|V\left(P \backslash\left\{v_{i}\right\}\right)\right|=6 \quad \text { npu } \quad i^{*}\left(P \backslash\left\{v_{i}\right\}\right)=5,
$$

где $i=1,2,3$.

Доказательство. Каждый $(x, y)$-разделитель множества $P=P(3,9,6)$ может быть либо $(5,3)$-разделителем, либо $(4,4)$-разделителем. Предположим, что $P$ имеет $(5,3)$-разделитель. (В случае, когда все $(x, y)$-разделители множества $P$ являются $(4,4)$-разделителями, результат не изменится.)

Пусть точка $u \in I(P)$ такая, что $u$ является $(5,3)$-разделителем множества $P$, где

$$
i^{*}\left(\triangle u v_{2} v_{3}\right)=5 \quad \text { и } \quad i^{*}\left(\triangle v_{1} u v_{3}\right)=3 .
$$

Если существует внутренняя точка $p \in I^{*}\left(\triangle v_{1} u v_{3}\right)$, лежащая в $H\left(v_{2} u ; v_{1}\right)$, то $i^{*}\left(\triangle p v_{2} v_{3}\right)=6$ или 7 , или 8. Итак, три внутренних точки треугольника $\triangle v_{1} u v_{3}$ лежат в $H\left(v_{2} u ; v_{3}\right)$. Тогда известно, что $\left|V\left(P \backslash\left\{v_{1}\right\}\right)\right|=6$ при $i^{*}\left(P \backslash\left\{v_{1}\right\}\right)=5$, поскольку иначе $i^{*}\left(P \backslash\left\{v_{1}\right\}\right)=6$ или 7 , или 8. Предположим, что

$$
V\left(P \backslash\left\{v_{1}\right\}\right)=\left\{u, v_{2}, v_{3}, w, x_{1}, x_{2}\right\} .
$$

Тогда $w$ является либо $(5,3)$-разделителем, либо $(4,4)$-разделителем множества $P$. Если $\left|V\left(P \backslash\left\{v_{3}\right\}\right)\right|=7$ или 8, то $i^{*}\left(P \backslash\left\{v_{2}\right\}\right)=6$ или 7, или 8. Если $\left|V\left(P \backslash\left\{v_{3}\right\}\right)\right|=3$ или 4, или 5, то $i^{*}\left(P \backslash\left\{v_{3}\right\}\right)=6$ или 7, или 8. Итак, $\left|V\left(P \backslash\left\{v_{3}\right\}\right)\right|=6$ при $i^{*}\left(P \backslash\left\{v_{3}\right\}\right)=5$. Ясно, что $\left|V\left(P \backslash\left\{v_{2}\right\}\right)\right|=6$ при $i^{*}\left(P \backslash\left\{v_{2}\right\}\right)=5$ (см. рис. 2 при $\left.k=6\right)$. 
Из существования множества $P(3,9,6)$ следует, что $t(6) \geqslant 10$. Чтобы доказать, что $t(6) \leqslant 10$, нам нужно доказать, что для любого точечного множества $P$ при $|I(P)| \geqslant 10$ всегда существует подмножество $Q \subset P$ при $i^{*}(Q)=6$ или 7 или 8 . Для краткости в последующих доказательствах всегда предполагается, что всякий треугольник $\triangle$ при $i^{*}(\triangle)=9$ является дефицитным точечным множеством $P(3,9,6)$, а $(x, y)$-разделители не рассматриваются при $x, y=6$ или 7 или 8 , поскольку иначе сразу получается искомое подмножество.

Лемма 3. Если $10 \leqslant|I(P)| \leqslant 15$ u $|V(P)|=3$, то существует подмножество

$$
Q \subset P \quad \text { при } \quad i^{*}(Q)=6 \text { или 7, или } 8 .
$$

ДокАЗАтЕльство. Пусть $V(P)=\left\{v_{1}, v_{2}, v_{3}\right\}$. Приведем доказательство для $|I(P)|=10$ (в случае $11 \leqslant|I(P)| \leqslant 15$ доказательства аналогичны).

Случай 1: множество $P$ имеет $(9,0)$-разделитель. Предположим, что $u_{1}$ является $(9,0)$ разделителем, где $i^{*}\left(\triangle u_{1} v_{2} v_{3}\right)=9$. Напомним, что $\triangle u_{1} v_{2} v_{3}$ - это дефицитное точечное множество $P(3,9,6)$. Пусть точка $u_{2} \in I^{*}\left(\triangle u_{1} v_{2} v_{3}\right)$ такая, что $i^{*}\left(\triangle u_{2} v_{2} v_{3}\right)=0$. Тогда без ограничения общности можно считать, что $u_{2} \in H\left(v_{1} u_{1} ; v_{3}\right)$. Применяя лемму 2 к треугольнику $\triangle u_{1} v_{2} v_{3}$, получим, что $u_{2}$ является вершиной треугольника $\triangle u_{1} v_{2} v_{3} \backslash\left\{v_{3}\right\}$. Предположим, что

$$
V\left(\triangle u_{1} v_{2} v_{3} \backslash\left\{v_{3}\right\}\right)=\left\{v_{2}, u_{2}, u_{3}, x_{1}, x_{2}, u_{1}\right\} .
$$

Если $u_{2}-(9,0)$-разделитель множества $P$, то $u_{3} \in H\left(v_{1} u_{2} ; v_{2}\right)$ и $i^{*}\left(\triangle v_{1} v_{2} u_{3}\right)=6$ или 7 , или 8. Если $u_{2}-(5,4)$-разделитель множества $P$, то $u_{3} \in H\left(v_{1} u_{2} ; v_{3}\right)$ и $i^{*}\left(P \backslash\left\{v_{3}\right\}\right)=6$ или 7, или 8 .

Случай 2: всякий $(x, y)$-разделитель множества $P$ является $(5,4)$-разделителем. Предположим, что $u_{1}-(5,4)$-разделитель, где $i^{*}\left(\triangle u_{1} v_{2} v_{3}\right)=5$ и $i^{*}\left(\triangle v_{1} u_{1} v_{3}\right)=4$. Тогда $i^{*}\left(P \backslash\left\{v_{1}\right\}\right)=5$ при $\left|V\left(P \backslash\left\{v_{1}\right\}\right)\right|=7$, поскольку иначе $i^{*}\left(P \backslash\left\{v_{1}\right\}\right)=6$ или 7 , или 8 . Кроме того, $i^{*}\left(P \backslash\left\{v_{2}\right\}\right)=4$ или 5 при $\left|V\left(P \backslash\left\{v_{2}\right\}\right)\right|=8$ или 7 , поскольку иначе $i^{*}\left(P \backslash\left\{v_{2}\right\}\right)=6$ или 7 , или 8 . Теперь легко определить подмножество, содержащее искомые внутренние точки.

Лемма 4. Если

$$
10 \leqslant|I(P)| \leqslant 13 \quad \text { u } \quad m=|V(P)| \geqslant 4,
$$

то существует подмножество $Q \subset P$ при $i^{*}(Q)=6$ или 7, или 8.

ДокАЗАТЕЛьство. Приведем простое доказательство равенства $|I(P)|=10$ (в случае $11 \leqslant|I(P)| \leqslant 13$ доказательства аналогичны).

Триангулируем множество $P$, т.е. соединим вершину $v_{1}$ выпуклой оболочки с каждой из остальных вершин выпуклой оболочки, и получим $m-2$ треугольника. Если существует треугольник $\triangle$ при $i^{*}(\triangle)=6,7,8$ или 10, то наше утверждение выполнено. Если существует треугольник $\triangle$ при $i^{*}(\triangle)=9$, то из леммы 2 следует, что искомое подмножество легко может быть найдено. Если существует треугольник $\triangle$ при $i^{*}(\triangle) \leqslant 4$, то желаемый результат легко получить, объединяя несколько смежных треугольников. Наконец, рассмотрим случай, когда существует треугольник $\triangle$ при $i^{*}(\triangle)=5$. Ясно, что в этом случае легко можно определить подмножество, содержащее 6 или 7 или 8 внутренних точек.

Нам также понадобится следующая лемма.

Лемма 5 [1]. Пусть $P$ - плоское точечное множество, имеющее $m \geqslant 3$ вершин и содержащее $s \geqslant 0$ внутренних точек. Тогда существует вершина $v_{i}$ множества $P$ такая, что множество $P^{\prime}=P \backslash\left\{v_{i}\right\}$ имеет $m^{\prime}$ вершин и содержит $s^{\prime}$ внутренних точек $u$

(a) либо $m^{\prime}=m-1 u s^{\prime}=s$,

(b) либо $m^{\prime}=m+t u s^{\prime}=s-t-1$, где $t=0,1,2, \ldots,\lfloor s / m\rfloor$. 
Лемма 6. Если $|V(P)| \geqslant 4$ и $|I(P)|=14$ или 15 , то существует подмножество $Q \subset P$ при $i^{*}(Q)=6$ или 7 или 8.

ДокАзАтЕЛЬство. Поскольку $|V(P)| \geqslant 4$, из леммы 5 следует существование собственного подмножества $P^{\prime} \subset P$ при $10 \leqslant i^{*}\left(P^{\prime}\right) \leqslant i(P)$ :

- если условие (а) выполняется, то $i^{*}\left(P^{\prime}\right)=13$ или 14 ;

- если условие (b) выполняется, то $i^{*}\left(P^{\prime}\right)=|I(P)|-\left\lfloor\frac{|I(P)|}{4}\right\rfloor-1 \geqslant 10$.

Тогда, применяя предыдущие результаты, получим подмножество $Q \subset P^{\prime} \subset P$ при $i^{*}(Q)=6$ или 7 , или 8 .

Аналогично доказывается следующий результат.

Лемма 7. Если $|V(P)| \geqslant 3 u|I(P)| \geqslant 16$, то существует подмножество $Q \subset P$ при $i^{*}(Q)=6$ или 7, или 8 .

Теперь неравенство $t(6) \leqslant 10$ следует из предыдущих результатов, и доказательство для случая $t(6)=10$ завершено.

\section{СПИСОК ЦИТИРОВАННОЙ ЛИТЕРАТУРЫ}

[1] D. Avis, K. Hosono and M. Urabe, Discrete Math., 241:1-3 (2001), 33-40. [2] T. Fevens, Discrete and Computational Geometry, Lecture Notes in Comput. Sci., 2866, Springer-Verlag, Berlin, 2003, 152-158. [3] Хианглин Вей, Рен Динг, "О плоских точечных подмножествах с заданным числом внутренних точек", Матем. заметки, 83:5 (2008), 752-756. [4] X. Wei, R. Ding, Surveys on Discrete and Computational Geometry: Twenty Years Later, Contemp. Math., 453, Amer. Math. Soc., Providence, RI, 2008, 515-528. [5] X. Wei, R. Ding, Discrete Comput. Geom., 42:4 (2009), 640-653. [6] D. Avis, K. Hosono, M. Urabe, Discrete and Computational Geometry, Lecture Notes in Comput. Sci., 1763, Springer-Verlag, Berlin, 2000, $57-64$.

\section{Хианлин Вей}

Поступило

Hebei University of Science and Technology, Китай

05.04.2011

E-mail: wxlhebtu@126.com 